\title{
Comparing short versions of the Alcohol Use Disorders Identification Test (AUDIT) in a military cohort
}

\author{
Jason Watterson, ${ }^{1,2,3,4}$ B Gabbe, ${ }^{1}$ P Dietze, ${ }^{1,5}$ A Bowring, ${ }^{5,6}$ J V Rosenfeld ${ }^{7,8,9}$
}

\begin{abstract}
- Additional material is published online only. To view please visit the journal online (http://dx.doi.org/10.1136/ jramc-2018-001024)

${ }^{1}$ Department of Epidemiology and Preventive Medicine, School of Public Health and Preventive Medicine, Monash University,

Melbourne, Australia ${ }^{2}$ National Trauma Research Institute, Alfred Health, Melbourne, Australia ${ }^{3}$ Royal Australian Navy, Canberra, Australia ${ }^{4}$ Department of Intensive Care and Hyperbaric Medicine, Alfred Health, Melbourne, Australia ${ }^{5}$ Behaviours and Health Risks Programs, Burnet Institute, Melbourne, Australia ${ }^{6}$ Department of Epidemiology, John Hopkins Bloomberg Schoo of Public Health, Baltimore, Maryland, USA

${ }^{7}$ Department of Neurosurgery, Alfred Hospital, Melbourne, Australia

${ }^{8}$ Australian Army, Canberra, Australia

${ }^{9}$ Department of Surgery, F. Edward Hebert School of Medicine, Uniformed Services University of Health Sciences, Bethesda, Maryland, USA
\end{abstract}

\section{Correspondence to} Jason Watterson, Alfred Hospital, Melbourne 3004, Victoria, Australia; jason.watterson@ monash.edu

Received 18 July 2018 Revised 15 September 2018 Accepted 17 September 2018 Published Online First 18 October 2018

Check for updates

(C) Author(s) (or their employer(s)) 2019. No commercial re-use. See rights and permissions. Published by BMJ.

To cite: Watterson J, Gabbe B, Dietze P, et al. I R Army Med Corps 2019:165:312-316.

\section{ABSTRACT}

Background The Alcohol Use Disorders Identification Test (AUDIT) is widely used for monitoring harmful alcohol consumption among high-risk populations. A number of short versions of AUDIT have been developed for use in time-constrained settings. In military populations, a range of AUDIT variations have been used, but the optimal combination of AUDIT items has not been determined.

Methods A total of 952 participants (80\% male), recruited as part of a wider study, completed the AUDIT10. We systematically assessed all possible combinations of three or four AUDIT items and established AUDIT variations using the following statistics: Cronbach's alpha (internal consistency), variance explained $\left(R^{2}\right)$ and Pearson's correlation coefficient (concurrent validity).

Results Median AUDIT-10 score was 7 for males and 6 for females, and 380 (40\%) participants were classified as having a score indicative of harmful or hazardous alcohol use $(\geq 8)$ according to WHO classifications.

A novel four-item AUDIT variation (3, 4, 8 and 9) performed consistently higher than established variations across statistical measures; it explained $85 \%$ of variance in AUDIT-10, had a Pearson's correlation of 0.92 and Cronbach's alpha was 0.63 . The FAST, an established shortened AUDIT variant, together with several other fouritem novel variants of AUDIT-10 performed similarly. The AUDIT-C performed consistently low on all measures, but with a satisfactory level of internal consistency $(75 \%)$.

Conclusion Shortened AUDIT variations may be suitable alternatives to the full AUDIT for screening hazardous alcohol consumption in military populations. Four-item AUDIT variations focused on short-term risky drinking and its consequences performed better than three item versions.

Trial registration number ACTRN12614001332617.

Hazardous or harmful alcohol consumption is a major public health issue in the Australian population, including in the Australian Defence Force (ADF). ${ }^{1}$ As a consequence, some ADF members experience elevated incidence of accidents, physical injury and other short-term harms as well as long-term health conditions and alcohol dependence. $^{2-4}$ Therefore, monitoring alcohol consumption is important to identify those at risk of harm along with consumption trends. This information is needed to inform, target and evaluate harm reduction strategies. ${ }^{5}$

The Alcohol Use Disorders Identification Test (AUDIT) was developed as a simple alcohol screening tool for the early identification of

\section{Key messages}

The 10-item Alcohol Use Disorders Identification Test (AUDIT) is widely used for monitoring harmful alcohol consumption among high-risk populations.

- Short versions of AUDIT have been developed for time-constrained settings, but the optimal combination of AUDIT items is unstudied in military populations.

- We systematically assessed all possible combinations of three or four item AUDIT variations.

- Each of the established and novel variations was systematically assessed using Cronbach's alpha (internal consistency), variance explained $\left(R^{2}\right)$ and Pearson's correlation coefficient (concurrent validity).

- Shortened AUDIT variations may be suitable alternatives to the full AUDIT for screening for hazardous alcohol consumption in military populations.

hazardous and harmful drinking, rather than identification of people who meet the criteria for alcohol dependence. ${ }^{6}$ The AUDIT has been widely used, including in military populations. ${ }^{7-9}$ The full AUDIT (AUDIT-10) comprises 10 items that span alcohol consumption, harms and dependence domains. However, a number of short versions of AUDIT have been developed for use in time-constrained settings, ${ }^{5}$ including the AUDIT-C (three items) and the FAST (four items). Of the shortened version, previously only the AUDIT-C has been used in the military setting, and this was primarily in the veteran community ${ }^{10-20}$ These, and other variants, have been shown to provide comparable information to the full AUDIT. For example, Bowring and colleagues, found that a four-item variant captured sufficient information about the consumption, dependence and harms domains to be suitable for routine screening of young people. ${ }^{5}$

The purpose of this study was to see if similar shortened versions of the AUDIT could prove useful in the screening of military personnel; minimising the number of items used will increase efficiency and allow measurement of other health behaviours which may be particularly important at the time of entry into military service. Items of the AUDIT used to develop a shortened version are likely to be population-specific, therefore we sought to 
identify the optimal combination of three to four AUDIT items for screening hazardous or harmful alcohol consumption in a sample of military recruits.

\section{METHODS}

\section{Setting and participants}

Initial Entry Trainees, aged 18-30 years, were recruited at the Royal Australian Navy's (RAN) key training establishment in Victoria, Australia between March 2014 and May 2016 as part of a randomised controlled trial (RCT), the protocol for which is published elsewhere. ${ }^{21}$ Once trainees complete basic training, they go on to complete their specialist training within one of several training schools of the ADF. Trainees were eligible to participate if their specialist training was for a minimum period of 13 weeks. During the enrolment period for this RCT, 2163 trainees were assessed for eligibility, 1211 did not meet the criteria and two trainees declined to participate. The remaining 952 trainees completed the baseline screening which included the AUDIT-10 prior to randomisation. Participation in the study was voluntary, and written informed consent was obtained from all participants.

\section{Registration}

The trial was registered with the Australian and New Zealand Clinical Trials Registry (ANZCTR): ACTRN12614001332617, date of registration: 18/12/2014 'retrospectively registered'.

\section{Questionnaires administered}

Each participant was asked to complete a baseline screening questionnaire which included demographic information (age, gender, faculty) and the AUDIT-10. Responses to the AUDIT-10 were based on the preceding 12 months for each participant. The questions of the AUDIT-10 can be divided into three domains: alcohol use (Q1-3), alcohol dependence (Q4-6) and adverse consequences of alcohol use (Q7-10) (table 1). The AUDIT is scored on a scale from 0 to $40 .^{22}$

\section{Analysis}

Data were entered into a REDCap database and statistical analysis was conducted using Stata V.12. ${ }^{23}$ The AUDIT-10 and established shortened versions of the AUDIT were scored (table 1). The AUDIT-10 scores were further classified and indicator variables were created to describe hazardous drinking $(\geq 8)$, high level of alcohol problems $(\geq 16)$ and possible alcohol dependence $(\geq 20)$ categories according to WHO recommended thresholds. $^{22}$

We assessed all possible combinations of three and four item AUDIT scales. Following a similar method to Bowring and colleagues, we required the tested shortened versions to cover all three domains of the AUDIT-10 (alcohol consumption, C; alcohol dependence, $\mathrm{D}$ and harmful alcohol use, $\mathrm{H}$ ) and include item 9 on alcohol related injury or be a recognised AUDIT variation with a maximum four items (AUDIT-3, AUDIT-C, FAST, AUDIT-4). ${ }^{5}$ Inclusion of item 9 was considered reasonable as military recruits are typically younger males ${ }^{24}$ and this item, focused on alcohol-related injury, has been shown to be important in previous studies of young people. ${ }^{25}$

The novel and established shortened versions of the AUDIT-10 used in the analysis are outlined in online supplementary appendix table.

Three measures were used to assess each three and four item combinations of the AUDIT, and the previously developed AUDIT variations. These measures were:

1. Cronbach's alpha: calculated to measure internal consistency of each AUDIT version, with a value of 0.7 or greater taken to indicate satisfactory reliability. ${ }^{6}$

2. $\mathrm{R}^{2}$ statistic: calculated from linear regression between individual items or novel combinations and the total AUDIT score to measure the total variance explained in the overall AUDIT-10 score by each individual item and novel combination of items.

3. Pearson's correlation coefficient: to examine the concurrent validity between each abbreviated AUDIT version.

Table 1 The AUDIT-10 questions and item inclusion in common established shortened AUDIT versions

\begin{tabular}{|c|c|c|c|c|c|c|c|}
\hline \multirow[b]{2}{*}{ Item no. } & \multirow[b]{2}{*}{ The AUDIT question } & \multirow{2}{*}{$\begin{array}{l}\text { AUDIT } \\
\text { domain* }\end{array}$} & \multicolumn{5}{|c|}{ Established AUDIT Variations } \\
\hline & & & AUDIT-10 & AUDIT- C & AUDIT-3 & FAST & AUDIT-4 \\
\hline 1 & How often do you have a drink containing alcohol? & $\mathrm{C}$ & $\cdot$ & $\cdot$ & & & - \\
\hline 2 & $\begin{array}{l}\text { How many drinks containing alcohol do you have on a typical } \\
\text { day when you are drinking? }\end{array}$ & $\mathrm{C}$ & $\cdot$ & $\cdot$ & & & $\cdot$ \\
\hline 3 & How often do you have six or more drinks on one occasion? & $\mathrm{C}$ & $\cdot$ & $\cdot$ & $\cdot$ & $\cdot$ & $\cdot$ \\
\hline 4 & $\begin{array}{l}\text { How often during the last year have you found that you were } \\
\text { not able to stop drinking once you had started? }\end{array}$ & $\mathrm{D}$ & $\cdot$ & & & & \\
\hline 5 & $\begin{array}{l}\text { How often during the last year have you failed to do what was } \\
\text { expected of you because of alcohol? }\end{array}$ & D & $\cdot$ & & & $\cdot$ & \\
\hline 6 & $\begin{array}{l}\text { How often during the last year have you needed a first drink } \\
\text { in the morning to get yourself going after a heavy drinking } \\
\text { session? }\end{array}$ & D & $\cdot$ & & & & \\
\hline 7 & $\begin{array}{l}\text { How often during the last year have you had a feeling of guilt } \\
\text { and remorse after drinking? }\end{array}$ & $\mathrm{H}$ & $\cdot$ & & & & \\
\hline 8 & $\begin{array}{l}\text { How often during the last year have you been unable to } \\
\text { remember what happened the night before because of your } \\
\text { drinking? }\end{array}$ & $\mathrm{H}$ & - & & & - & \\
\hline 9 & $\begin{array}{l}\text { Have you or someone else been injured because of your } \\
\text { drinking? }\end{array}$ & $\mathrm{H}$ & $\cdot$ & & & & \\
\hline \multirow[t]{2}{*}{10} & $\begin{array}{l}\text { Has a relative, friend, doctor or other healthcare worker been } \\
\text { concerned about your drinking or suggested you cut down? }\end{array}$ & $\mathrm{H}$ & $\cdot$ & & & $\cdot$ & $\cdot$ \\
\hline & & Score range: & $0-40$ & $1-12$ & $0-4$ & $0-16$ & $1-16$ \\
\hline
\end{tabular}

${ }^{*}$ AUDIT domains are: $\mathrm{C}$, alcohol consumption; $\mathrm{D}$, alcohol dependence; $\mathrm{H}$, harmful alcohol use. ${ }^{5}$ 
Table 2 Statistical scores for novel and established shortened AUDIT variations, ordered from highest to lowest overall rank

\begin{tabular}{|c|c|c|c|c|c|}
\hline Overall rank & $\begin{array}{l}\text { Selected novel and established } \\
\text { combinations }\end{array}$ & $\begin{array}{l}\text { AUDIT } \\
\text { Domain* }\end{array}$ & $\begin{array}{l}\text { Explained variance of total } \\
\text { AUDIT-10 score }\left(R^{2}\right)\end{array}$ & Cronbach's alpha & Pearson's correlations $(r)$ \\
\hline 1 & $3,4,8$ and 9 & $C, D, H$ & 0.85 & 0.63 & 0.92 \\
\hline 2 & $3,5,8$ and 9 & $C, D, H$ & 0.84 & 0.63 & 0.92 \\
\hline 4 & FAST $(3,5,8$ and 10$)$ & $C, D, H$ & 0.83 & 0.61 & 0.91 \\
\hline 5 & $2,3,5$ and 9 & $C, D, H$ & 0.83 & 0.61 & 0.91 \\
\hline 8 & $3,6,8$ and 9 & $C, D, H$ & 0.81 & 0.63 & 0.90 \\
\hline 9 & $2,5,8$ and 9 & $C, D, H$ & 0.82 & 0.60 & 0.91 \\
\hline 10 & $2,3,6$ and 9 & $C, D, H$ & 0.81 & 0.60 & 0.90 \\
\hline 18 & AUDIT-4 $(1,2,3$ and 10$)$ & $\mathrm{C}, \mathrm{H}$ & 0.74 & 0.66 & 0.86 \\
\hline 30 & AUDIT-C (1, 2 and 3$)$ & C & 0.65 & 0.75 & 0.80 \\
\hline 50 & 2,6 and 9 & $C, D, H$ & 0.70 & 0.41 & 0.90 \\
\hline 52 & 1,4 and 9 & $C, D, H$ & 0.70 & 0.38 & 0.92 \\
\hline 53 & 1,5 and 9 & $C, D, H$ & 0.66 & 0.42 & 0.90 \\
\hline 54 & 1,6 and 9 & $C, D, H$ & 0.61 & 0.41 & 0.92 \\
\hline 55 & AUDIT- 3 (3) & $C$ & 0.54 & 0.44 & 0.73 \\
\hline
\end{tabular}

*AUDIT domains are: $\mathrm{C}$, alcohol consumption; $\mathrm{D}$, alcohol dependence; $\mathrm{H}$, harmful alcohol use. ${ }^{5}$

Performance on each of these statistics was ranked from highest to lowest. The sum of the ranks of three statistics was then calculated and the abbreviated AUDIT versions were re-ranked based on the sum of all ranks. ${ }^{5}$

\section{RESULTS}

\section{Demographic profile}

There were 952 participants; the median (IQR) age was 20.4 (19.1-22.9) years and 80\% were male. The median (IQR) AUDIT-10 score for males was 7 (5-9) and 6 (4-9) for females. Of the total sample, 36\% $(n=343)$ were classified as hazardous drinkers (score $\geq 8), 2 \%(n=21)$ had high-level alcohol problems (score $\geq 16)$ and $1.7 \%(n=16)$ were possibly alcohol dependent (score $\geq 20$ ). The distribution of drinking behaviours did not differ by gender $(p=0.11)$.

There were 330 possible three-item or four-item combinations. Only $51(15 \%)$ combinations met the specified criteria of representing three domains and containing item 9. The rankings of all 51 combinations, and the existing AUDIT variations, are shown in the (online supplementary appendix table). The 20 highest-ranked three-item and four-item novel combinations as well as the results for the four pre-existing variations, are shown in table 2 . The highest ranked overall across all three analyses was the novel combination of $3,4,8$ and 9 . In addition to the injury item, this combination includes information on items largely related to the practice and consequences of short-term risky drinking.

\section{Cronbach's alpha (internal consistency)}

The internal consistency of the AUDIT-10 in our sample was 0.80 . The highest internal consistency of the three-item combinations was obtained with the AUDIT-C (0.75), followed by the novel combination of items 3,5 and $9(0.5)$ (table 2). This finding is not surprising given that the three-items of the AUDIT-C all come from the same (consumption) domain; inclusion of the key injury item reduced internal consistency considerably. The highest internal consistency of the four-item combinations was obtained with two of the novel combinations $3,4,8$ and 9 and 3, 5, 8 and 9 (0.63). The FAST (0.61) ranked fourth overall and was the highest ranked of the established four-item combinations.

\section{Variance explained}

Of the three-item combinations the AUDIT-C explained the most variance $(65 \%)$ in the AUDIT-10 score, followed by the combination of items 1,6 and 9 which explained $61 \%$ (table 2). This finding is consistent with previous research showing that consumption explains a large proportion of the variance in AUDIT scores in general population studies. Of all four-item combinations, the combination of items $3,4,8$ and 9 explained the most variance (85\%) in the AUDIT-10 score while the combination of items 2, 3, 6 and 9 explained $81 \%$ and the AUDIT- 4 explained 74\% of the variance in AUDIT-10 score. The novel combination of 2, 3, 6 and 9 includes item 6 clearly related to dependence and a marker of risky drinking for long term harm.

\section{Pearson's correlation coefficient (concurrent validity)}

Most of the combinations shown in table 2 demonstrated high levels of concurrent validity with $17 / 20$ correlating with the AUDIT-10 at 0.90 or above. Correlation with the AUDIT-10 was highest with the four-item combinations (each 0.92): 3, 4, 8 and 9; 3, 5, 8 and 9 and 2, 3, 4 and 9. All remaining novel four-item and three-item combinations also demonstrated correlations of at least 0.90 . The FAST demonstrated the highest correlation with the AUDIT-10 $(\rho=0.91)$ of the established short versions. The correlations between the AUDIT-10 and AUDIT-4 $(\rho=0.86)$, and the AUDIT-C $(\rho=0.80)$ were lower. 


\section{DISCUSSION}

We assessed combinations of AUDIT items to determine an optimal shortened version of the questionnaire for use in screening military recruits. Our results align with previous work with different populations showing that much of the information available from the full AUDIT scale can be captured using just a few items of the AUDIT-10. ${ }^{52627}$ We found that the novel combinations 3,4,8 and 9;3, 5, 8 and 9 and 2, 3, 4 and 9 out-ranked the FAST, which was the highest ranked four-item combination in previous work with young people. ${ }^{5}$ However, we included item 9 on alcohol-related injury as a key AUDIT item for young populations such as active military personnel. Given these novel combinations outperform the FAST and include alcohol-related injury, we suggest that they are appropriate for use with military personnel. However, it should be noted that the differences in the key statistics were relatively small across the top-10 ranked combinations meaning that all of these could be considered for screening purposes. This means that screeners can select from combinations on the basis of specific items of interest. For example, in contexts where the consequences of long-term risky drinking are of most interest, then a combination including the dependence item 6 may be important to include, while item 4 may be most important where the consequences of risky drinking for short term harm are the focus.

Importantly, the AUDIT consumption items included in all of the top 10 combinations related to frequency of short-term risky drinking and/or quantity typically drunk rather than frequency of drinking (item 1), and the top three combinations all included the frequency of short-term risky drinking item. This finding suggests the focus of screening and intervention with military recruits is best directed towards this short-term risky drinking pattern, consistent with previous reviews and work in the area. ${ }^{101824}$

In previous studies of military populations, the AUDIT-C has been the most commonly used AUDIT-10 variant. While the AUDIT-C was the only abbreviated variant demonstrating satisfactory internal consistency, this result stems from the fact that all three items come from a single domain. As the AUDIT-C performed below other variants on other measures, we argue that preferentially including questions from other domains of harm and dependence provides a more reliable measure as a replacement for the full AUDIT-10 when screening for alcohol consumption and harm in military populations.

There are a number of limitations to this study and these should be considered when interpreting the findings. As noted in similar studies exploring shortened AUDIT versions, we did not include a gold standard or comparative clinical diagnosis of harmful/hazardous alcohol use or alcohol-related problems, such as the DSM-IV, precluding assessment of predictive validity. ${ }^{526}$ Consequently, we were only able to determine the performance of the novel AUDIT variations in comparison with the AUDIT-10, which itself has not been validated in this military population. Further, the shortened AUDIT models were not independent of the AUDIT-10, and thus our comparison violated the assumption of an independence for assessing linear regression (variance explained). While this method has been used in previous related studies, our findings should still be interpreted with appropriate caution. ${ }^{28}$ Finally, our study involved a large sample of largely young naval recruits and the appropriateness of direct extrapolation of the findings to age groups and other military settings should be considered.

\section{CONCLUSION}

Among a sample of young military personnel, novel four-item combinations that included items largely related to shortterm risky drinking and its consequences showed the highest internal consistency, variance and concurrent validity when compared with the full AUDIT-10. Our findings suggest these novel combinations could be viable alternatives for screening for alcohol misuse in large military populations. While three of the novel combinations tested ranked the highest across the three statistics measured, any of the variations within the top 10 appear to be as effective as the AUDIT-10 at screening for hazardous or harmful alcohol consumption in this population, allowing fine-grained selection of combinations to suit different contexts. Further testing of these novel versions against an independent measure of hazardous alcohol consumption, such as captured using the DSM-IV, is required to establish the best shortened version for use in the military setting.

Acknowledgements The authors would like to acknowledge the participants of the study, along with their instructors and supervisors who enabled their participation. We thank the command team of HMAS Cerberus for their support of this programme. We also thank the P.A.R.T.Y. programme team and the team at the NTRI for their contributions to the programme.

Contributors JW, BG, PD and $A B$ designed the study. JW wrote the first draft of this paper. $B G$ and $P D$ revised the first draft and all authors contributed to successive drafts. All authors read and approved the final manuscript.

Funding This project was funded by a Defence Health Foundation grant. BG was supported by a National Health and Medical Research Council of Australia Career Development Fellowship (GNT1048731) during the preparation of this manuscript.

Declarations of competing interest JW, BG, AB and JVR declare that they have no competing interests. PD has received an untied educational grant from Reckitt Benckinser and an investigator-driven grant from Gilead Sciences for work unrelated to this paper.

Ethics approval Departments of Defence and Veterans' Affairs HREC, Alfred Health HREC and Monash University HREC.

Provenance and peer review Not commissioned; externally peer reviewed.

\section{REFERENCES}

1 Australian Defence Force. 2014. Australian Defence Force Alcohol Management Strategy and Plan 2014-2017. Canberra: Commonwealth of Australia.

2 Bonomo YA, Bowes G, Coffey C, et al. Teenage drinking and the onset of alcohol dependence: a cohort study over seven years. Addiction 2004;99:1520-8.

3 Livingston M, Room R. Variations by age and sex in alcohol-related problematic behaviour per drinking volume and heavier drinking occasion. Drug Alcohol Depend 2009;101:169-75.

4 NHMRC. Australian Guidelines to reduce health risks from Drinking Alcohol. Canberra: Department of Health and Ageing, 2009.

5 Bowring AL, Gouillou M, Hellard M, et al. Comparing short versions of the AUDIT in a community-based survey of young people. BMC Public Health 2013;13:301.

6 Saunders JB, Aasland OG, Babor TF, et al. Development of the alcohol use disorders identification test (AUDIT): WHO collaborative project on early detection of persons with harmful alcohol consumption--II. Addiction 1993;88:791-804.

7 Kelsall HL, Wijesinghe MS, Creamer MC, et al. Alcohol use and substance use disorders in Gulf War, Afghanistan, and Iraq War veterans compared with nondeployed military personnel. Epidemiol Rev 2015;37:38-54.

8 McFarlane A, Hodson S, Van Hooff M. 2010. Mental health in the Australian defence force: 2010 ADF mental health and wellbeing study: full report. Canberra: Commonwealth of Australia.

9 Waller M, McGuire AC, Dobson AJ. Alcohol use in the military: associations with health and wellbeing. Subst Abuse Treat Prev Policy 2015;10:27.

10 Aguirre M, Greenberg N, Sharpley J, et al. Alcohol consumption in the UK armed forces: are we drinking too much? J R Army Med Corps 2014;160:72-3.

11 Bradley KA, Lapham GT, Hawkins EJ, et al. Quality concerns with routine alcohol screening in VA clinical settings. J Gen Intern Med 2011;26:299-306.

12 Bradley KA, Williams EC, Achtmeyer CE, et al. Implementation of evidence-based alcohol screening in the Veterans Health Administration. Am J Manag Care 2006;12:597-606

13 Bradley KA, Rubinsky AD, Lapham GT, et al. Predictive validity of clinical AUDIT-C alcohol screening scores and changes in scores for three objective 
alcohol-related outcomes in a Veterans Affairs population. Addiction 2016;111:1975-84.

14 Calhoun PS, Wagner HR, Wagner HR. The prevalence of binge drinking and receipt of provider drinking advice among US veterans with military service in Iraq or Afghanistan. Am J Drug Alcohol Abuse 2015;50:1-10.

15 Delaney KE, Lee AK, Lapham GT, et al. Inconsistencies between alcohol screening results based on AUDIT-C scores and reported drinking on the AUDIT-C questions: prevalence in two US national samples. Addict Sci Clin Pract 2014:9:2.

16 Fuehrlein BS, Mota N, Arias AJ, et al. The burden of alcohol use disorders in US military veterans: results from the National Health and Resilience in Veterans Study. Addiction 2016;111:1786-94.

17 Harris AH, Lembke A, Henderson P, et al. Risk of future trauma based on alcohol screening scores: a two-year prospective cohort study among US veterans. Addict Sci Clin Pract 2012;7:6.

18 Harbertson J, Hale BR, Watkins EY, et al. Pre-deployment alcohol misuse among shipboard active-duty u.s. military personnel. Am J Prev Med 2016;51:185-94.

19 Possemato K, Funderburk J, Spinola S, et al. Reliability and Validity of a treatment barriers scale for individuals with alcohol use disorder. Subst Use Misuse 2016:51:383-94.

20 Williams EC, Frasco MA, Jacobson IG, et al. Risk factors for relapse to problem drinking among current and former US military personnel: a prospective study of the Millennium Cohort. Drug Alcohol Depend 2015;148:93-101.
21 Watterson J, Gabbe B, Dietze P, et al. Measuring the effectiveness of in-hospital and on-base Prevent Alcohol and Risk-related Trauma in Youth (P.A.R.T.Y.) programs on reducing alcohol related harms in naval trainees: P.A.R.T.Y. Defence study protocol. BMC Public Health 2017:17.

22 Babor T, Higgins-Biddle J, Saunders J, et al. The alcohol use disorders identification test: guidelines for use in primary care. 2nd edn. Geneva: World Health Organization, Department of Mental Health and Substance Dependence, 2001.

23 StataCorp. Stata Statistical Software: Release 12. College Station, TX: StataCorp LP, 2012.

24 Hamilton M. The Use of Alcohol in the Australian Defence Force. Report of the independant advisory panel on alcohol. Canberra: Commonwealth of Australia, 2011.

25 Chikritzhs TN, Pascal R. Trends in Youth Alcohol Consumption and Related Harms in Australian Jurisdictions, 1990-2002, National Alcohol Indicators: Bulletin No 6. Perth, Australia: National Drug Research Institute, 2004.

26 Aalto M, Alho $\mathrm{H}, \mathrm{Halme}$ JT, et al. AUDIT and its abbreviated versions in detecting heavy and binge drinking in a general population survey. Drug Alcohol Depend 2009:103(1-2):25-9.

27 Cortés-Tomás M-T, Giménez-Costa J-A, Motos-Sellés P. Different versions of the alcohol use disorders identification test (audit) as screening instruments for underage binge drinking. Drug Alcohol Depend 2016;158:52-9.

28 McCambridge J, Thomas BA. Short forms of the AUDIT in a Web-based study of young drinkers. Drug Alcohol Rev 2009;28:18-24. 